\title{
Algoritmo particle swarm optimization (PSO) no treinamento de redes neurais para estimativa de altura em povoamento de eucalipto
}

\author{
Harliany de Brito Matias ${ }^{1}$, Daniel Henrique Breda Binoti ${ }^{1}$, Gilson Fernandes da Silva ${ }^{2}$, Helio Garcia \\ Leite $^{3}$
}

\footnotetext{
${ }^{1}$ Programa de Pós-Graduação em Ciências Florestais, Universidade Federal do Espírito Santo (UFES), Jerônimo Monteiro, ES, Brasil.

${ }^{2}$ Departamento de Engenharia Florestal, Universidade Federal do Espírito Santo (UFES), Jerônimo Monteiro, ES, Brasil. ${ }^{3}$ Departamento de Engenharia Florestal, Universidade Federal de Viçosa (UFV), Viçosa, MG, Brasil.
}

RESUMO O presente trabalho objetivou avaliar o ajuste do algoritmo Particle Swarm Optimization (PSO) no treinamento de RNA para estimativa de altura de árvores de eucalipto. Os dados utilizados neste estudo foram provenientes de medições de inventários florestais contínuos conduzidos em povoamentos de um clone de eucalipto, localizados no Sul da Bahia, Brasil. A altura das árvores foi estimada em função das seguintes variáveis numéricas (quantitativas): diâmetro com casca a 1,3 m, altura dominante média da parcela em $(\mathrm{m})$, idade em meses, altura média das parcelas em $(\mathrm{m})$, dap médio das parcelas em (cm) e dap máximo das parcelas em $(\mathrm{cm})$. Enquanto que para a variável qualitativa foram: projeto e rotação. O algoritmo PSO, nas suas cinco configurações, apresentou uma melhor estimativa de altura quando comparado ao modelo hipsométrico ajustado. Porém suas estimativas não foram tão satisfatórias quando comparado com configurações tradicionais utilizadas em RNA, no caso do algoritmo Resilient propagation, em sua variação básica (RPROP+). Tanto as redes treinadas pelo PSO quanto aquelas treinadas pelo Resilient são mais eficientes para estimação da altura das árvores, do que o modelo de regressão testado neste estudo.

Palavras-chave: relação hipsométrica; Metaheuristica; inteligência artificial.

\section{Algorithm particle swarm optimization (PSO) in neural network training for height estimate in settlement}

\begin{abstract}
This study aimed to evaluate the fit of the Particle Swarm Optimization algorithm (PSO) in RNA training to estimate eucalyptus trees tall. The data used in this study were derived from measurements of continuous forest inventories conducted in stands of eucalyptus clone, located in southern Bahia, Brazil. The height of the trees was estimated according to the following numerical variables (quantitative): diameter with bark $1.3 \mathrm{~m}$, average dominant height of the parcel (m), age in months, average height of the plots $(\mathrm{m})$, dap average the plots in $(\mathrm{cm})$ and dap most of the plots in $(\mathrm{cm})$. While for the qualitative variable were: Design and rotation. The PSO algorithm in its five configurations presented a better estimate of the time when compared to the adjusted hypsometric model. However, their estimates were not so satisfactory compared to traditional configurations used in RNA in the case of Resilient propagation algorithm in its basic variation (Rprop + ). Both networks trained by PSO as those trainadas by Resilient are more efficient to estimate the height of the trees, than the regression model tested in this study.
\end{abstract}

Keywords: hypsometric relationship; metaheuristic; artificial intelligence.

\section{Introdução}

Para o planejamento florestal hierárquico é necessário obter informações qualitativas e quantitativas dos povoamentos.
Algumas dessas informações, muitas vezes são obtidas por meio de procedimentos de estimação. Exemplo disso, é a obtenção da altura estimada das árvores, uma vez que a medição da altura de todas as árvores é inviável, devido ao excessivo 
tempo e custo. Nesse contexto, o setor florestal sempre busca por procedimentos de estimação cada vez mais exatos (MARTINS et al., 2014) e menor esforço na coleta e processamento de dados.

A altura das árvores é necessária para estimar o volume de madeira, classificação da capacidade produtiva e modelagem do crescimento e da produção, sendo esse volume fundamental na fase de planejamento da produção para qualquer empresa florestal (RIBEIRO et al., 2010).

A predição da altura das árvores por regressão usualmente é feita incluindo variáveis explicativas como o dap (diâmetro a 1,3 m de altura), a altura dominante $(H d)$, a idade (I) e a densidade $(N)$. Os modelos empregados podem ser lineares ou não lineares (CAMPOS e LEITE, 2013 e BATISTA et al., 2001).

Uma alternativa para estimar a altura de árvores em povoamentos equiâneos é a abordagem por redes neurais artificiais - RNA (BINOTI et al., 2013). Os excelentes resultados já obtidos por meio de redes neurais artificiais se devem as suas características intrínsecas que as tornam adequadas para solucionar diferentes problemas. As RNA possuem a habilidade para detectar implicitamente relações não-lineares entre a variável de saída e as variáveis de entrada, sendo essa uma característica interessante para muitas das estimativas florestais (ÖZÇELIK et al., 2010). Entretanto, a definição de uma configuração de RNA apropriada para solucionar um determinado problema é geralmente empírica e pode demandar muito tempo para encontrar a rede ideal, devido ao número elevado de combinações possíveis dos atributos das redes, tais como número de neurônios, função de ativação, algoritmos de aprendizagem e seus parâmetros (BRAGA et al., 2007; ARTERO, 2009).

Existem diferentes algoritmos de treinamento, como backpropagation Resilient, Quickprop, Skip-Layer, entre ou- tros. Na maioria das vezes, para estimação da altura de árvores, tem sido mais comum o emprego dos algoritmos backpropagation e resilient. Entretanto, o tipo de algoritmo utilizado interfere no tempo de treinamento e na eficiência de uma rede (BINOTI et al., 2014).

Um algoritmo que vem sendo aplicado com sucesso em muitas áreas como otimização de funções, treinamento de redes neurais artificiais, controle de sistemas de lógica Fuzzy, em planejamento Florestal é o Particle Swarm Optimization (PSO) (COELHO; WEIHMANN, 2007; NASCIMENTO et al, 2012). O qual segundo (KENNEDY e EBEHART 1995a, 1995b), possui vantagens como: (i) possui fácil implementação computacional, requerendo poucas linhas de código; (ii) utiliza pouca memória e requer pouca velocidade de processamento; e (iii) o processo de busca é racionalizado pelo contínuo aprendizado das partículas.

Este algoritmo foi apresentado em 1995 (KENNEDY; EBERHART, 1995). É uma técnica que se baseia no movimento coletivo de um grupo de partículas: o enxame de partículas. Cada membro deste enxame é movimentado através do espaço de busca do problema por duas forças. Uma os atrai com uma magnitude aleatória para a melhor localização já encontrada por ele próprio (pbest) e outra para a melhor localização encontrada entre alguns ou todos os membros do enxame (gbest). A posição e a velocidade de cada partícula são atualizadas a cada iteração até todo o enxame convergir obtendo o melhor resultado (CASTRO; TSUZUKI, 2007; NASCIMENTO et al, 2012).

Assim, o objetivo do presente estudo foi testar um novo processo de treinamento de RNA utilizando o algoritmo Particle Swarm Optimization para estimar a altura de árvores de eucalipto, bem como avaliar sua eficiência quando comparado ao método de treinamento clássico com o algoritmo $R e$ silient propagation e a metodologia tradicional (modelos hipsométricos). 


\section{Materiais e Métodos}

\section{Descrição dos dados}

Os dados utilizados neste estudo foram provenientes da medição de 3178 árvores em 323 parcelas de inventário instaladas e medidas em povoamentos de eucalipto, localizado no sul da Bahia, Brasil, em uma área de cerca de 2.000 ha. O espaçamento de plantio foi de $3 \times 3 \mathrm{~m}$ e a idade, no ano de medição, variou de 29 a 111 meses (Tabela 1).

A altura da árvore $i$ da parcela $j\left(H t_{\mathrm{ij}}\right)$ foi estimada em função das seguintes variáveis numéricas (quantitativas): diâmetro da árvore individual $i$ na parcela $j\left(d a p_{\mathrm{ij}}\right)$, altura dominante da parcela $j\left(H d_{j}\right)$, idade da árvore $i$ na parcela $j\left(I_{i j}\right)$, altura média da parcela $j\left(H_{j}\right)$, dap médio da parcela $j\left(\right.$ dapmed $\left._{j}\right)$, e dap máximo da parcela $j\left(\right.$ dapmax $\left._{j}\right)$. As variáveis categóricas consideradas no estudo foram nome do projeto e regime de corte.

Tabela 1. Análise descritiva das variáveis numéricas utilizadas na estimação da altura de árvores individuais

Table 1. Descriptive analysis of numerical variables used to estimate the height of individual trees

\begin{tabular}{ccccc}
\hline Variáveis & Mínimo & Máximo & Média & $\begin{array}{c}\text { Desvio } \\
\text { padrão }\end{array}$ \\
\hline$I_{i j}($ meses $)$ & 29 & 111 & 63 & $\pm 20,3$ \\
dap $_{\mathrm{ij}}(\mathrm{cm})$ & 5,1 & 31,2 & 16,2 & $\pm 4,7$ \\
$H d_{j}(\mathrm{~m})$ & 14,4 & 25,5 & 19,2 & $\pm 2,2$ \\
$H_{j}(\mathrm{~m})$ & 13,6 & 22,4 & 17,4 & $\pm 1,7$ \\
dapmed $_{j}(\mathrm{~cm})$ & 10,1 & 15,4 & 12,8 & $\pm 1,2$ \\
dapmax $_{j}(\mathrm{~cm})$ & 12,6 & 21,7 & 16,5 & $\pm 2,1$ \\
$\operatorname{Ht}_{\mathrm{ij}}(\mathrm{m})$ & 8,3 & 43,9 & 25,5 & $\pm 6,1$ \\
\hline
\end{tabular}

\section{Modelo Hipsométrico}

Para efeito de comparação com os resultados da aplicação de RNA, foi ajustado o modelo:

$$
L n H t_{i j}=\beta_{0}+\beta_{1} d a p_{i j}{ }^{-1}+\beta_{2} \ln H d j+\varepsilon_{i j}
$$

em que: $L n H t_{i j}=$ Logaritmo neperiano de altura total da árvore $i$ na parcela $j ; n H d_{j}=$ Logaritmo de altura dominante na parcela $j$; $d a p_{i j}=$ diâmetro a $1,3 \mathrm{~m}$ de altura, da árvore $i$ na parcela $j$. $\beta_{0}, \beta_{1}$ e $\beta_{2}=$ parâmetros do modelo; e $\varepsilon=$ erro.

\section{Redes Neurais Artificiais}

Algoritmo Resilient Propagation: Empregou-se como método de treinamento clássico o algoritmo Resilient propagation, na variação básica denominada RPROP (BRAGA et al., 2000). De acordo com estes autores, a principal diferença entre este algoritmo e as outras heurísticas baseadas em variações do "backpropagation" é que os ajuste dos pesos $(\omega)$ dos neurônios da rede e da taxa de aprendizado $(\eta)$ depende apenas dos sinais dos gradientes da função erro $E(\omega)$, não dependendo, portanto, de sua magnitude. A função $E(\omega)$ é responsável pela especificação de um critério de desempenho que está associado à rede.

No algoritmo "RPROP”, os pesos e a taxa de aprendizado são alterados apenas uma única vez em cada época de treinamento. Cada peso $\omega_{j i}$ possui sua própria taxa de variação $\left(\Delta_{j i}\right)$, a qual varia em função do tempo $t$ da seguinte forma:

$$
\begin{aligned}
& \Delta_{j i}(t) \\
& =\left\{\begin{array}{l}
\eta^{+} \cdot \Delta_{j i}(t-1), \text { se } \frac{\partial E}{\partial \omega_{j i}}(t-1) \cdot \frac{\partial E}{\partial \omega_{j i}}>0 \\
\eta^{-} \cdot \Delta_{j i}(t-1), \text { se } \frac{\partial E}{\partial \omega_{j i}}(t-1) \cdot \frac{\partial E}{\partial \omega_{j i}}<0 \\
\Delta_{j i}(t-1), \\
\text { caso contrário }
\end{array}\right.
\end{aligned}
$$

Em que: $0<\eta^{-}<1<\eta^{+}$.

Uma mudança no sinal das derivadas parciais correspondentes ao peso $\omega_{j i}$ indica que a última mudança foi grande suficiente para que o sistema saltasse sobre um ponto de mínimo da função $E(\omega)$, o que implica então em uma diminuição do valor de $\Delta_{j i}$ proporcional ao fator $\eta^{-}$. Já as derivadas consecutivas com o mesmo sinal indicam que o sistema está movendo permanentemente em uma única direção, o que implica assim em um aumento sensível de $\Delta_{j i}$ proporcional ao fator $\eta^{+}$.

Os pesos da rede são então alterados através das seguintes equações: 


$$
\Delta \omega_{j i}(t)=\left\{\begin{array}{l}
-\Delta_{j i}(t), \text { se } \frac{\partial E}{\partial \omega_{j i}}(t)>0 \\
+\Delta_{j i}(t), \text { se } \frac{\partial E}{\partial \omega_{j i}}(t)<0 \\
0, \quad \text { caso contrário }
\end{array}\right.
$$

O valor de atualização $\Delta_{j i}$ é definido por um processo de adaptação que depende do sinal da derivada do erro com relação ao peso a ser ajustado.

Algoritmo Particle Swarm Optimization: como metodologia testada foi empregado a metaheurística Particle Swarm Optimization (PSO), que é baseada no movimento coletivo de um grupo de partículas, o enxame de partículas. Cada partícula deste enxame é movimentada através do espaço de busca do problema por duas forças. Uma os atrai, com uma magnitude aleatória, para a melhor localização já encontrada por ele próprio (pbest) e outra para a melhor localização encontrada entre alguns ou todos os membros do enxame (gbest). A posição e a velocidade de cada partícula são atualizadas a cada repetição até todo o enxame convergir (CASTRO; TSUZUKI, 2007).

No mecanismo de funcionamento do PSO, como observado na Figura 1, inicia-se cada partícula com valores aleatórios de posição e velocidade. Durante a sua execução cada partícula avaliará sua solução atual em relação à melhor posição já encontrada por ela mesma, fazendo com que o valor de pbest seja atualizado. Cada partícula também avaliará a qualidade da melhor solução encontrada na sua vizinhança, sendo o valor de gbest atualizado também (NASCIMENTO et al, 2012).

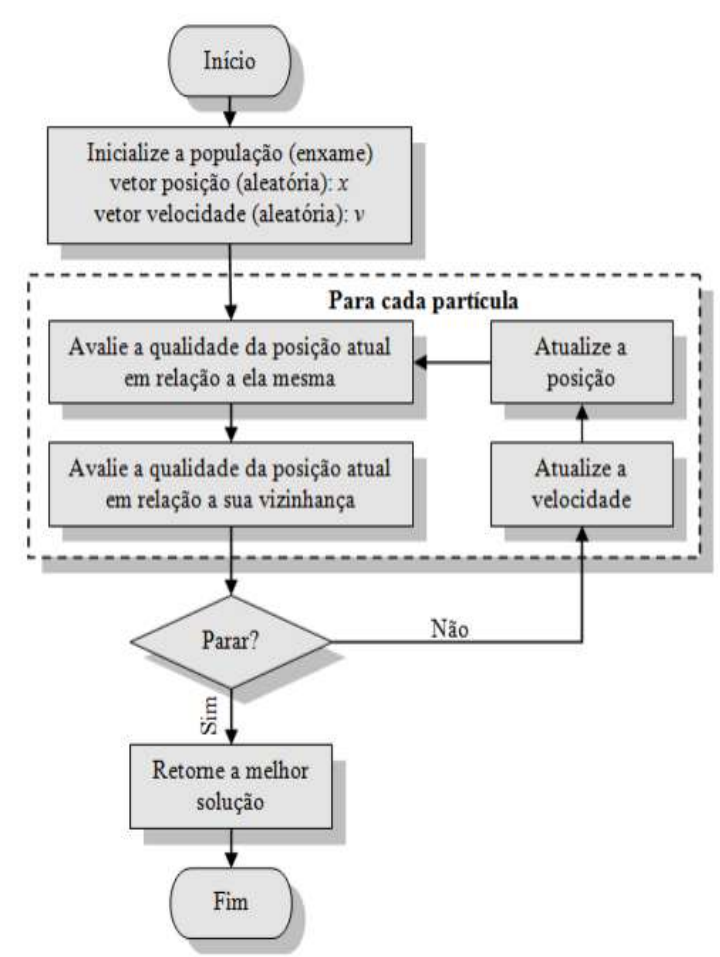

Figura 1. Fluxograma demonstrando o funcionamento do algoritmo PSO (Fonte: Nascimento et al., 2012).

Figure 1. Flowchart showing the operation of the PSO algorithm (Source: Nascimento et al, 2012).

\section{Treinamento das Redes e Avaliação das esti-}

\section{mativas}

Para o treinamento das RNA utilizou-se o sistema Neuro 4, o qual está disponibilizado gratuitamente na homepage http://neuroforest.ucoz.com (BINOTI, 2012). Foram testadas 30 redes do tipo Multilayer Perceptron (MLP) com uma camada intermediária de 8 neurônios, sendo 5 RNA para o algoritmo Resilient propagation e 5 para cada configuração do algoritmo PSO. A função de ativação utilizada foi a logística. As variáveis de entrada e saída foram padronizadas entre [0, 1]. Foi empregado como critério de parada 3.000 épocas para cada combinação de configuração. Para o Algoritmo Resilient propagation empregou-se como método de treinamento a variação básica denominada RPROP. Para o Algoritmo (PSO) foram treinadas diferentes tamanhos de populações $(50,100$, 200, 500 e 1000) afim de verificar qual configuração do algoritmo obteria um melhor resultado para estimativa de altura. 
As estimativas de altura obtidas com a aplicação das RNA foram avaliadas com base nas estatísticas de correlação entre os valores estimados e observados das alturas projetadas e a raiz quadrada do erro quadrático médio (RMSE\%) equação (4) e equação (5), conforme Binoti et al. (2011).

$$
r_{Y \hat{Y}}=\frac{\operatorname{cov}(Y, \hat{Y})}{\sqrt{s^{2}(Y) s^{2}(\hat{Y})}}
$$

em que: $s^{2}=$ variância; $\operatorname{cov}=$ covariância; $\hat{y}=$ Estimativa da variável dependente; e y = variável dependente.

$$
\operatorname{RMSE}(\%)=100 \sqrt{\frac{\sum_{i=1}^{n}(Y-\hat{Y})}{n}} / \bar{Y}
$$

em que: $\bar{Y}=$ média da saída observada (altura); $\mathrm{n}=$ número total de dados; $\hat{y}=$ Estimativa da variável dependente; e y = variável dependente.

\section{Resultados e Discussão}

O coeficiente de correlação entre os valores observados e estimados pela equação de altura foi 0,7690. As estimativas apresentaram-se tendenciosas, com superestimação nos indivíduos inferiores e superiores, em termos de dap. A relação entre alturas observadas e alturas estimadas, a dispersão dos erros relativos percentuais em funções dos valores observados e a distribuição de frequência dos erros relativos percentuais são apresentados na Figura 2.

As alturas estimadas pelas RNA resultaram em tendência semelhante para as diferentes parametrizações do algoritmo PSO: população inicial de 50, 100, 200, 500 e 1000 e para o algoritmo Resilient propagation (Figura 3). Ao analisar os gráficos de resíduos, observa-se que as alturas estimadas pelas redes apresentaram uma tendência de subestimação nos menores valores de diâmetro.

De forma complementar, foi analisado graficamente a distribuição dos erros relativos percentuais.
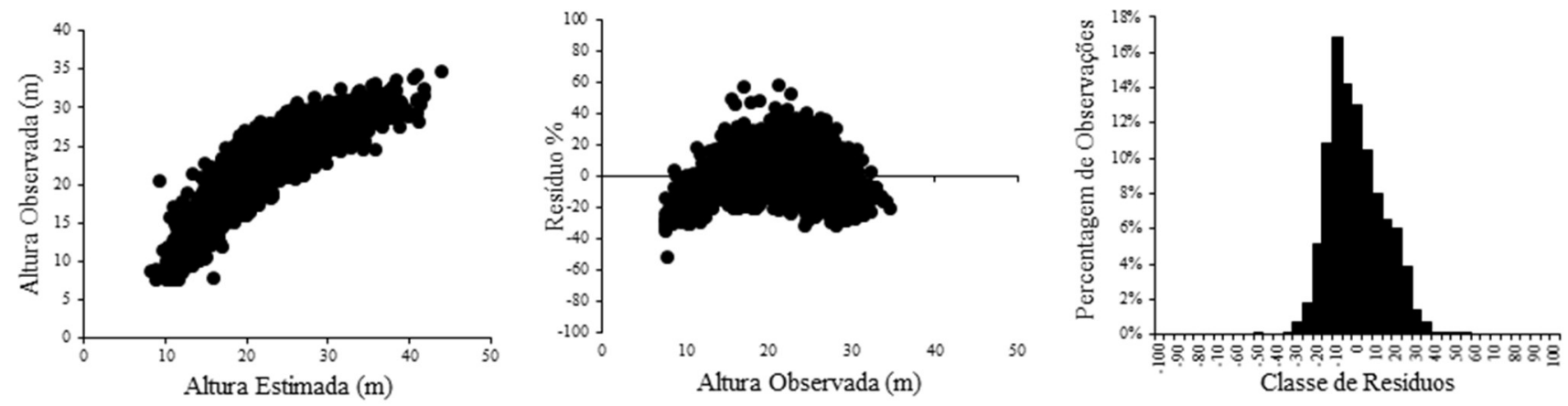

Figura 2. Relação entre alturas observadas e alturas estimadas, dispersão dos erros relativos percentuais em funções das alturas observadas e a distribuição de frequência dos erros relativos percentuais das estimativas obtidas por regressão.

Figure 2. Relationship between observed and estimated heights, dispersion of percentage relative errors in the observed heights functions and the frequency distribution of the percentage errors in the estimates obtained by regression. 

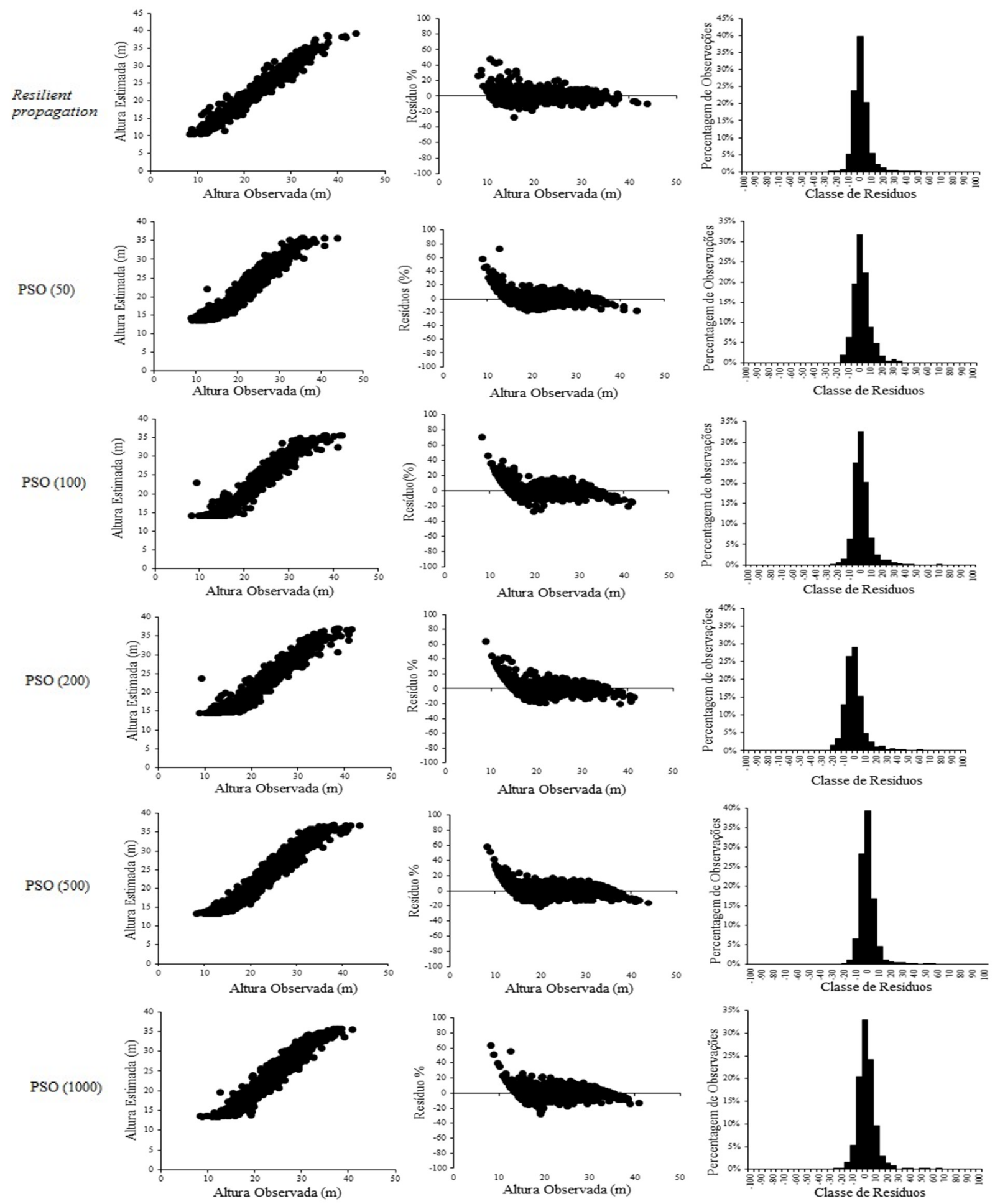

Figura 3. Relação entre alturas observadas e alturas estimadas, dispersão dos erros relativos percentuais em funções das alturas observadas e a distribuição de frequência dos erros relativos percentuais das estimativas obtidas com RNA treinadas pelo algoritmo PSO.

Figure 3. Relationship between observed and estimated heights heights, dispersion of percentage relative errors in functions of the observed heights and the frequency distribution of the percentage errors in the estimates obtained with RNA trained by PSO algorithm. 
O algoritmo PSO, nas suas cinco configurações, apresentou uma melhor estimativa de altura quando comparado ao modelo hipsométrico ajustado (Figura 2). Porém suas estimativas não foram tão satisfatórias quando comparado com configurações tradicionais utilizadas em RNA, no caso do algoritmo resilient propagation, em sua variação básica (RPROP+). Isso também pode ser observado a partir dos valores dos coeficientes de correlação apresentados na Tabela 2.

Os resultados obtidos para as alturas de eucalipto estimadas por RNA, utilizando o algoritmo particle swarm optimization (PSO), demonstram que essa metodologia pode ser utilizada e apresentam boas estimativas, porém não superou a eficiência do algoritmo resilient propagation. Indica-se então realizar novos estudos testando diferentes configurações do algoritmo afim de obter melhores resultados.
Segundo Silva et al. (2009), a obtenção da arquitetura adequada da rede neural depende de inúmeras tentativas, a fim de gerar resultados satisfatórios, uma vez que esse processo é aleatório, ou seja, o número de neurônios por camadas não se baseia em nenhum critério, apenas em tentativa.

De acordo com Martins et al. (2014), os excelentes resultados alcançados por meio de RNA se devem as suas características intrínsecas que as tornam adequadas para solucionar diferentes problemas. As RNA possuem a habilidade para detectar implicitamente relações não-lineares entre a variável de saída e as variáveis de entrada, sendo essa uma característica interessante para muitas das estimativas florestais.

Tabela 2. Coeficiente de correlação para o modelo hipsométrico ajustado e as diferentes parametrizações das RNA treinadas. Table 2. Correlation coefficient for the adjusted hypsometric model and different parameterization of trained RNA.

\begin{tabular}{ccccccc}
\hline \multicolumn{7}{c}{ Coeficiente de correlação } \\
\hline Modelo hipsométrico & Resilient propagation & PSO (50) & PSO (100) & PSO (200) & PSO (500) & PSO (1000) \\
\hline 0,769009 & 0,985012 & 0,978921 & 0,963637 & 0,962636 & 0,971082 & 0,968584 \\
\hline
\end{tabular}

\section{Conclusões}

A estimativa de altura de árvores de eucalipto por meio de redes neurais artificiais, ajustada pelo algoritmo Particle Swarm Optimization (PSO), não resulta em maior exatidão quando comparada à redes treinadas pelo Resilient $\left(\mathrm{RPROD}^{+}\right)$. Tanto as redes treinadas pelo PSO quanto aquelas treinadas pelo Resilient são mais eficientes para estimação da altura das árvores, do que o modelo de regressão testado neste estudo.

\section{Referências}

ARTERO, A. O. Inteligência Artificial: teórica e prática. 1 ed. São Paulo: Editora Livraria da Física, 2009. 230p.
BATISTA, J. L. F.; COUTO, H. T. Z.; MARQUESINI, M. Desempenho de modelos de relações hipsométricas: estudo em três tipos de florestas. Scientia Forestalis, n. 60, p. 149-163, 2001.

BRAGA, A. P.; LUDERMIR, T. B.; CARVALHO, A. P. de L. F. Redes neurais artificiais: teoria e aplicações. Rio de Janeiro: Livros Técnicos e Científicos, 2000. 262p.

BRAGA, A. de P.; CARVALHO, A. C. P. de L. F. de; LUDEMIR, T. B. Redes Neurais Artificiais: teoria e aplicações. 2. ed. Rio de Janeiro: LTC, 2007. 226 p.

BINOTI, D. H. B.; BINOTI, M. L. M. S.; LEITE, H. G.; GARCIA, S. L. R.; CRUZ, J. P. Modelos de distribuição diamétrica para povoamentos desbastados de eucalipto utilizando a função hiperbólica. Revista Árvore, Viçosa-MG, v. 35, n. 5, p. 1069-1076, 2011. 
BINOTI, D. H. B. Sistemas computacionais aplicados ao manejo florestal. 2012. 122 p. Tese. (Doutorado em Ciência Florestal) - Universidade Federal de Viçosa, Viçosa, 2012.

BINOTI, M. L. M. S.; BINOTI, D. H. B.; LEITE, H. G. Aplicação de redes neurais artificiais para estimação da altura de povoamentos equiâneos de eucalipto. Revista Árvore, ViçosaMG, v. 37, n. 4, p. 639-645, 2013.

BINOTI, D. H. B.; BINOTI, M. L. M. S.; LEITE, H. G. Configuração de redes neurais artificiais para estimação do volume de árvores. Ciência da Madeira, Pelotas, v. 5, n. 1, p. 58-67, 2014.

CAMPOS, J. C. C.; LEITE, H. G. Mensuração florestal: perguntas e respostas. 4. ed. Viçosa: UFV, 2013. 548 p.

CASTRO, E.G.; TSUZUKI, M.S.G. Simulation optimization using swarm intelligence as tool for cooperation strategy design in 3d predator-prey game. In: CHAN, F.T.S.; TIWARI, M.K. Swarm intelligence - focus on ant and particle swarm optimization. Viena: I-Tech Education and Publishing, 2007. $532 \mathrm{p}$.

COELHO, L. S.; WEIHMANN, L. Abordagem de enxame de partículas inspirada em algoritmo cultural aplicada em um problema de otimização não-linear com restrições. In: Sociedade Brasileira de Automática - I Simpósio Brasileiro Inteligência Computacional, Florianópolis, SC, 2007.

KENNEDY, J.; EBERHART, R. C. Particle swarm optimization. In: IEEE INTERNATIONAL CONFERENCE ON NEURAL NETWORKS, Perth, Austrália, 1995. Proceedings... Perth: IEEE, 1995. p. 1942-1948.

KENNEDY, J.; EBERHART, R. C. A new optimizer using particle swarm theory. In: PROCEEDINGS OF THE 6TH INTERNATIONAL SYMPOSIUM ON MICROMACHINE AND HUMAN SCIENCE, Nagoya, 1995b. Proceedings... Nagoya: IEEE, 1995b.

MARTINS, E. R.; ARAÚJO, A. C. S. C.; SILVA, P. V.; BINOTI, M. L. M. S.; BINOTI, D. H. B.; LEITE, H.G. Parametrização de Redes Neurais Artificiais para estimação da altura total de árvore de povoamento de eucalipto. Atualidades em mensuração florestal [livro eletrônico]. 1. Ed. 407p. 373376p. Curitiba, 2014.

NASCIMENTO, F. A. F.; DIAS, A. N.; FIGUEIREDO FILHO, A.; ARCE, J. E.; MIRANDA, G. M. Uso da Meta-Heurística otimização por exame de partículas no planejamento Florestal. Scientia Forestalis, Piracicaba, v. 40, n. 96, p. 557-565, dez. 2012.

ÖZÇELIK, R.; DIAMANTOPOULOU, M.J.; BROOKS, J.R.; WIANT Jr., H. Estimating tree bole volume using artificial neural network models for four species in Turkey. Journal of Environmental Management, v. 91, n. 3, p. 742-753, 2010.

RIBEIRO, A.; FERRAZ FILHO, A. C.; MELLO, J. M.; FERREIRA, M. Z.; LISBOA, P. M. M.; SCOLFORO, J. R. S. Estratégias e metodologias de ajuste de modelos hipsométricos em plantios de Eucalyptus sp. Cerne, Lavras, v. 16, n. 1, p. 22-31, jan./mar. 2010.

SILVA, M. L. M.; BINOTI, D. H. B.; GLERIANI, J. M.; LEITE, H. G. Ajuste do modelo de Schumacher e Hall e aplicação de redes neurais artificiais para estimar volume de árvores de eucalipto. Revista Árvore, v. 33, n. 6, p. 1133-1139, 2009. 